Bryan Roberts, BS, OMS III, Andrew E. Makar, BS, OMS III, Ryan Canaan, BA, OMS III, Vanessa Pazdernik, MS and Tatyana Kondrashova*, MD, PhD

\title{
Effect of occipitoatlantal decompression on cerebral blood flow dynamics as evaluated by Doppler ultrasonography
}

https://doi.org/10.1515/jom-2020-0100

Received April 22, 2020; accepted August 10, 2020;

published online February 12, 2021

\section{Abstract}

Context: Osteopathic manipulative treatment reduces symptoms in patients with headache disorders, but the underlying mechanisms are unclear.

Objective: To evaluate blood flow in the intracranial and extracranial vasculature before and after occipitoatlantal decompression (OAD) using Doppler ultrasonography.

Methods: Healthy, first-year osteopathic medical students from A.T. Still University’s Kirksville College of Osteopathic Medicine participated in a randomized, single-blinded, two-period, two-treatment crossover study. The participants were randomly assigned to 1 of 2 treatment interventions: OAD or sham touch. After one week, participants returned to have the other intervention performed. Blood flow parameters-peak systolic velocity (PSV) and end-diastolic velocity (EDV)-in the middle cerebral artery (MCA), internal carotid artery (ICA), and vertebral artery (VA) were evaluated before, immediately after, 5 minutes after, and 10 minutes after treatment. Differences in PSV, EDV, heart rate (HR), and blood pressure (BP) for both interventions were analyzed for the four time points using mixed-effects models.

Results: Thirty healthy medical students (11 men, 19 women; mean age, 24 years) participated in this study. EDV increased after OAD in the MCA, ICA, and VA (all p<0.001); no change occurred after sham touch (all $p>0.05$ ). EDV was

*Corresponding author: Tatyana Kondrashova, MD, PhD, Kirksville College of Osteopathic Medicine, A.T. Still University, 800 W. Jefferson St., Kirksville, MO, 63501-1443, USA, E-mail: tkondrashova@atsu.edu Bryan Roberts, BS, OMS III, Andrew E. Makar, BS, OMS III and Ryan Canaan, BA, OMS III, Kirksville College of Osteopathic Medicine, A.T. Still University, Kirksville, MO, USA

Vanessa Pazdernik, MS, Department of Research Support, A.T. Still University, Kirksville, MO, USA greater for all post-treatment timepoints after OAD in the MCA, ICA, and VA than after sham touch (all $\mathrm{p}<0.001$ ). Although baseline PSV in the MCA measured before treatment was different between treatment interventions ( $\mathrm{p}=0.01$ ), no difference was found between interventions at any post-treatment time point (all $\mathrm{p}>0.59$ ). Changes in PSV in the ICA and VA and for HR and BP did not depend on treatment intervention ( $\mathrm{p}>0.06)$.

Conclusion: Increases in EDV occurred in major cranial arteries after OAD but not after sham touch, indicating that OAD improves blood flow to the brain. The exact mechanism of this increase is unknown; however, it can be explained by either parasympathetic stimulation through the secretion of vasodilating neurotransmitters or by a decrease in external tissue pressure on ICA and VA, with the resulting flow causing further dilation in the MCA.

Keywords: Doppler sonography; headache; occipitoatlantal decompression; osteopathic manipulative treatment.

Occipitoatlantal decompression (OAD) is a cranial osteopathic manipulative treatment (OMT) technique used to free local restrictions around the occipitoatlantal joint. ${ }^{1-3}$ Similar techniques with slightly different force vectors are used in this anatomical area as well, including condylar decompression for the occipital condyles and suboccipital decompression for the area surrounding the suboccipital muscles. ${ }^{1-5}$ These techniques can be used to normalize parasympathetic tone, improve infant feeding issues, and manage headaches. ${ }^{1,3-6}$ In a previous heart rate variability study, ${ }^{6}$ researchers investigated the effect of suboccipital decompression on parasympathetic tone. Heart rate variability was significantly affected by suboccipital decompression, indicating a possible influence on the vagus nerve and the corresponding parasympathetic tone to the heart. ${ }^{6}$ The hypoglossal nerve may be influenced by OMT as well. ${ }^{4,5}$ When treated with condylar decompression, infants with feeding issues have shown improvements in their suckling response. ${ }^{4,5}$ In theory, this management technique releases

This work is licensed under the Creative Commons Attribution 4.0 
soft tissue restrictions (fascia, ligaments, muscle, and bone) around the condyles where the hypoglossal nerve travels, including the hypoglossal canal; these restrictions are thought to reduce proper neurotransmission to control tongue movements during feeding. ${ }^{4,5}$

Patients with headache disorders who visit osteopathic physicians may experience a reduction of symptoms when OMT is used as part of their treatment plan. ${ }^{7,8}$ For example, OAD is one of many techniques used to manage headache disorders, but little research has investigated the physiological effects of OAD, especially concerning headache conditions. Some studies ${ }^{9-11}$ have investigated using multiple cranial and cervical techniques to manage headaches. Overall, the current evidence for OMT and headache disorders is low, so more research is needed to determine whether these methods are effective and to understand why they are effective. ${ }^{12,13}$

Although pharmacological treatments exist for headache disorder prevention and reduction, they may limit the patient's quality of life and treatment outcomes because of associated risks, contraindications, expenses, and side effects such as worsening symptoms (e.g., chronic daily headache from overuse). ${ }^{14,15}$ Alternatively, OAD is a quick OMT technique that can be performed during an office visit. ${ }^{1-3,6,16}$ Further, it can be combined with other OMT techniques, such as soft tissue and inhibition techniques, or performed as a standalone osteopathic treatment. Although techniques like soft tissue can help relax muscle tension and relieve certain types of headache disorders (e.g., tension headaches), other types of headache disorders (e.g., migraines) are more complex with multifactorial etiology, symptomatology, and phases that involve extracranial and intracranial blood vessels, nerves, chemical imbalances, or inflammation, and can also be genetic or environmental. ${ }^{10}$ For migraines, evidence supports neural and vascular involvement. ${ }^{17,18}$ However, the vascular hypothesis is controversial because it is debated whether migraines originate from blood flow or neuronal changes. ${ }^{19,20}$ The trigeminovascular system is likely involved and widely accepted as contributing to the complex pathophysiology of the headache phase of migraines. ${ }^{21,22}$ Specifically, nociceptors in large intracranial vessels and dura mater are stimulated and send afferent pain signals to the trigeminal ganglia, which form the trigeminovascular system and contribute to the development of migraines. ${ }^{21,22}$ Vasoactive neuropeptides, such as calcitonin gene-related peptide, are released in the vascular terminals of the trigeminal nerve and its nucleus. ${ }^{23}$ Calcitonin gene-related peptide has been found to induce the release of proinflammatory mediators and may cause vasodilatory effects in cerebral and dural blood vessels. ${ }^{24,25}$ This results in additional activation of the afferent fibers of the trigeminal nerve, causing a positive feedback loop for further calcitonin gene-related peptide release. ${ }^{24,25}$ During the cortical spreading depression of the aura phase of migraine, slow depolarization waves and cortical inhibition occur around the same time as aura symptoms, such as visual or motor disturbances. ${ }^{26}$ Blood flow changes like cortical hyperemia and oligemia also take place during this phenomenon. ${ }^{26}$ A local cortical spreading depression has been suggested to release calcitonin gene-related peptide, potassium ions, glutamate, nitrous oxide, and other molecules that diffuse and activate the nociceptors involved in the trigeminovascular response observed during the headache phase of migraines. ${ }^{27,28}$

The clinical benefit of OMT for the management of headache disorders may arise from neurovascular effects, particularly when OAD is used. ${ }^{8,29}$ Other benefits of OAD are that it does not have as many side effects as medications and may be used as an adjunctive treatment in addition to medications. This kind of combined treatment approach may reduce the amount of medication used, provide immediate symptom relief, and improve the patient's quality of life.

Transcranial Doppler ultrasonography is a noninvasive tool that can be used to evaluate blood flow in the cerebral arteries. $^{30}$ In the clinical setting, transcranial Doppler ultrasonography has various applications and is especially useful in intensive care, since it can be used to evaluate intracranial stenosis, occlusion, and intracranial pressure. In patients with migraines, transcranial Doppler ultrasonography has shown significant changes in circulation during a headache when compared with headachefree periods. ${ }^{31}$ Therefore, it would be beneficial to use transcranial Doppler to assess possible vascular mechanisms by which OAD helps prevent or reduce headache disorders. Such information would allow physicians to better understand how to use this OMT technique most effectively whether in combination with other modalities or as a standalone therapy.

The purpose of the current study was to evaluate blood flow in the intracranial and extracranial vasculature before and after OAD using Doppler ultrasonography. We hypothesized that OAD would result in changes in blood flow to the head, which could provide a better understanding of the effects of OAD on cerebral vasculature and aid in the development of individualized treatment plans for patients with headache disorders.

\section{Methods}

Healthy, first-year osteopathic medical students from A.T. Still University's Kirksville College of Osteopathic Medicine 
were recruited to this randomized, single-blinded, twoperiod, two-treatment crossover study. First-year osteopathic medical students were sent a mass email inviting their participation in the study, and describing its purpose: to examine blood flow to the brain as a result of OMT. They were informed that Doppler ultrasonography would be used to evaluate blood flow in the internal carotid, vertebral, and middle cerebral arteries. They were provided an electronic sign-up form with available dates and times. Students who participated in the first portion of the study were invited back for the second portion of the study one week later. First-year students were chosen for the study because they had not yet been exposed to OAD in their didactics and skills labs at the time of data collection. Participation was voluntary, students were not compensated, and there was no penalty for not participating. No identifying information was collected during the study. Background information, including demographic characteristics and a brief medical history checklist, was collected from each participant. If any of the conditions listed on the medical history were present, the participant was excluded from the study. Conditions listed for exclusion were: caffeine use $\leq 2$ hours before participating in the data collection, rigorous exercise the day of the data collection, OMT the day of the data collection, smoking, cervical spinal injuries/fractures/trauma, fusion of cervical vertebra(e), vertebrobasilar artery insufficiency, cervical herniated disk, pregnancy, rheumatoid arthritis, chronic migraine (with and/or without aura), currently experiencing headache or migraine, severe osteoporosis, ligamentous instability, history of concussion, brain injuries, ocular trauma, vision disturbance, glaucoma, arterial hypertension, neoplasm, and Ehlers-Danlos, Marfan, or any other connective tissue disorder. All procedures of the current study were approved by the A.T. Still University-Kirksville institutional review board.

To evaluate blood flow in the intracranial and extracranial vasculature before and after OAD using Doppler ultrasonography, students participated in two periods and were randomly assigned to OAD or sham touch treatment interventions at the first period by a coin toss. Participants returned for the next period after one week and the second treatment intervention was performed. The randomization was used to control for intraparticipant variability. We included a sham touch intervention to compare OAD with the effect of simple skin touch. Participants were unaware of the type of treatment performed and were instructed not to have caffeine for at least 2 hours before the sessions.

Before each treatment intervention, the participant was placed supine on a treatment table for 5 minutes to stabilize blood pressure. ${ }^{32-34}$ After 5 minutes, the blood flow parameters of peak systolic velocity (PSV) and end-diastolic velocity (EDV) were measured in the middle cerebral artery (MCA), internal carotid artery (ICA), and vertebral artery (VA) using ultrasonography. PSV was used to evaluate systolic flow and EDV was used to evaluate diastolic flow. Each participant's heart rate (HR) and blood pressure (BP) were also measured to evaluate the systemic effect of OAD. These primary outcome variables were measured at four timepoints: before treatment (baseline), immediately after treatment, 5 minutes after treatment, and 10 minutes after treatment.

We measured blood flow parameters in both the intracranial (MCA) and extracranial (ICA and VA) arteries supplying the brain. The MCA is the terminal branch of the ICA, is the largest of the cerebral arteries, and supplies the lateral aspect of the cerebral cortex (motor and somatosensory to all but the lower extremity), the temporal pole, internal capsule, and basal ganglia. ${ }^{35}$ The ICA and the external carotid artery are terminal branches of the common carotid arteries. While the external carotid artery stays superficial and mostly supplies the superficial aspect of the head and neck, the ICA enters the cranium through the carotid canal of the temporal bone to supply the anterior and middle cerebral circulation, including the forebrain..$^{35,36}$ The VA is an arterial vessel that arises from the subclavian artery. It travels within the transverse foramina of the cervical vertebrae and enters the cranium through the foramen magnum. At that point, the VA pierces the dura and arachnoid mater, where it becomes intracranial and eventually combines to form the basilar artery. Including its branches, the basilar artery supplies the cerebellum and posterior cerebral circulation, such as the occipital lobe. ${ }^{35,36}$

For the OAD treatment, the operator (B.R.) sat at the head of the treatment table placed the pads of his index fingers on the areas slightly superolateral to the posterior tubercle of the Atlas of the participant and reinforced his index fingers with his middle fingers. Next, the operator moved his reinforced index fingers superoanteriorly to provide tension until firm contact with the occiput of the participant was established. The operator then applied lateral traction by supinating his wrist and forearm and moving his elbows medially (bringing his reinforced index fingers laterally) while simultaneously providing gentle traction on the occiput of the participant. This entire combination provided posterolateral distraction of the area. In practice, the technique is completed when the operator feels a release of tissue tension. ${ }^{1-3}$ The time for this release can vary between patients depending on the degree and severity of their dysfunction and the inherent viscoelastic properties of individual tissues. To standardize the technique while maintaining clinical relevance, we 
sought a consensus between clinical osteopathic research and clinical practice, which suggested that successful treatment occurred from around 30 seconds to up to $3 \mathrm{mi}-$ nutes. ${ }^{6,16}$ Thus, to appropriately monitor all variables during treatment in the current study, 2 minutes was determined as the standard time to hold the treatment position for all participants. After 2 minutes of $\mathrm{OAD}^{6,16}$ the operator slowly guided the participant back to a neutral head position. At that time, the primary outcome variables were measured and recorded by other operators (A.M., R.C., T.K.). The participant remained in the supine position for 10 minutes after treatment, and all measurements were repeated at 5 and 10 minutes after treatment.

For the sham touch treatment, the protocol was similar to the OAD treatment, and primary outcome variables were measured at the same timepoints. However, during the sham treatment, the operator's index and middle fingerpads lightly touched the participant's occiput, around the suboccipital sulcus, for 2 minutes. There was no lateral traction, anterior tension, or firm contact. This protocol prevented the operator from applying any posterolateral distraction force to the area.

Cerebral blood flow in the MCA was evaluated unilaterally with transcranial Doppler ultrasonography using a Mindray-5 ultrasound machine (Shenzhen Mindray BioMedical Electronics, Co.) with a $2 \mathrm{MHz}$ phased array transducer. With the participants in the supine position, a standard scanning technique was used through the temporal window, located above the zygomatic ridge between the lateral canthus of the eye and the auricular pinna. The ICA and VA blood flow was assessed unilaterally using a 10-12 MHz linear probe using color and pulsed-wave Doppler. The ICA was insonated in its proximal part, near the bifurcation. The VA was evaluated at the C3-C5 level. The participant's HR and BP were recorded simultaneously with the vascular blood flow measurements. HR was measured using the pulsed-wave Doppler waveform of the ICA and an automatic BP cuff. BP was measured using an automatic BP cuff. A physician certified by the American
Registry for Diagnostic Medical Sonography with 19 years of ultrasonography and teaching experience performed all ultrasonography (T.K.).

Linear mixed-effects models were used to assess the degree to which changes over time depended on treatment for PSV, EDV, HR, and BP. Baseline measurements were included as additional response variables with accompanying fixed treatment effects to allow for distinct estimates. We controlled for period effects unique to baseline and post-treatment timepoints. The model included an unstructured covariance matrix for measurements from the same student and period and an additional unstructured covariance matrix between baseline and post-treatment measurements on the same student across both periods. This allowed responses at each timepoint to not be constrained by the same variance and allowed for correlation among measurements from the same person. We summarized the model results using the estimated mean at each timepoint for each treatment with corresponding standard errors (SE) or 95\% confidence intervals (CI) and estimated mean change from baseline at each post-treatment timepoint. SAS version 9.4 statistical software (SAS Institute, Inc.) was used to conduct the analyses. A value of $p<0.05$ was considered statistically significant.

\section{Results}

Thirty healthy participants (19 women, 11 men; mean age, 24 years) took part in the study. Mean body mass index was 23.4 and $67 \%$ (20 of 30 ) had a body mass index less than 25 . Half (15 of 30) of the students received OAD first and then sham touch. The remaining students received sham touch first and then OAD.

The EDV showed an interaction between time and treatment in the MCA, ICA, and VA (all p<0.001). EDV increased over time after OAD in the MCA, ICA, and VA (all $\mathrm{p}<0.001$ ); no changes occurred after sham touch (all p $>0.05$; Figures 1, 2, and Table 1). EDV at all post-treatment

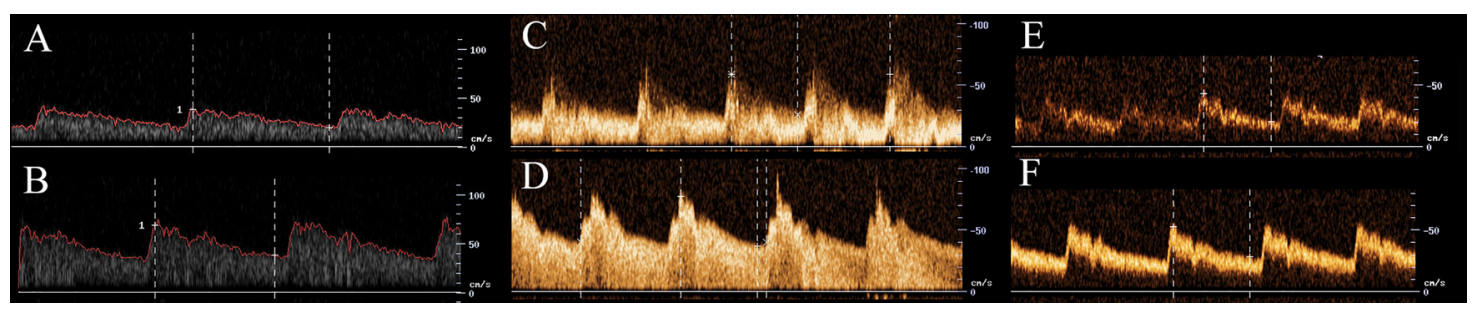

Figure 1: Pulsed-wave Doppler ultrasonography images of intracranial and extracranial vessels before and after occipitoatlantal decompression (OAD): (A) middle cerebral artery (MCA) before OAD, (B) MCA after OAD, (C) internal carotid artery (ICA) before OAD, (D) ICA after $O A D,(E)$ vertebral artery (VA) before OAD, (F) VA after OAD. 

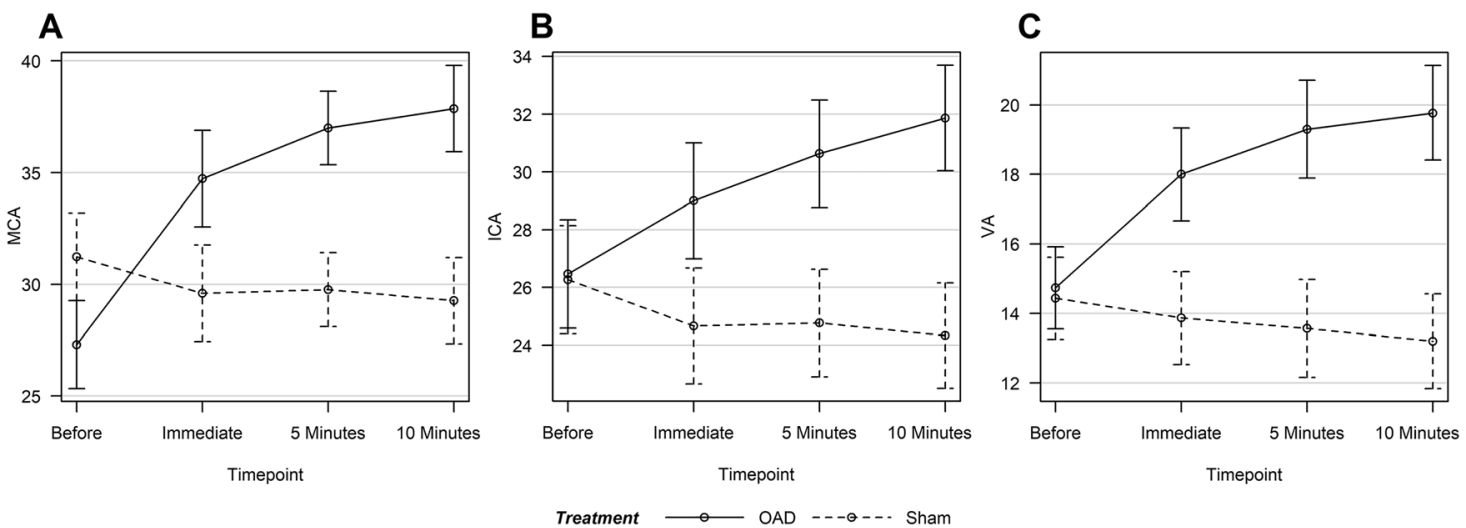

Figure 2: End-diastolic velocity (EDV) in intracranial and extracranial vessels at different study timepoints before and after occipitoatlantal decompression (OAD) or sham touch treatment: (A) middle cerebral artery (MCA), (B) internal carotid artery (ICA), (C) vertebral artery (VA). Error bars represent $95 \%$ confidence intervals.

Before = baseline measurements before the study intervention, Immediate = measurements taken immediately after the study intervention, 5 minutes $=$ measurements taken 5 minutes after the study intervention, and 10 minutes = measurements taken 10 minutes after the study intervention.

Table 1: Interaction of time and treatment of arteries and blood flow after management with occipitoatlantal decompression and sham treatment.

\begin{tabular}{|c|c|c|c|c|c|c|c|c|c|c|}
\hline \multirow[t]{2}{*}{ Artery } & \multirow{2}{*}{$\begin{array}{l}\text { Blood } \\
\text { flow }\end{array}$} & \multirow[t]{2}{*}{ Timepoint } & \multicolumn{3}{|r|}{ OAD } & \multicolumn{3}{|r|}{ Sham } & \multirow{2}{*}{$\begin{array}{r}\text { Mean: OAD } \\
\text { vs. sham } \\
\text { p-Value }\end{array}$} & \multirow{2}{*}{$\begin{array}{l}\text { Change from } \\
\text { baseline: } O A D \text { vs. sham }\end{array}$} \\
\hline & & & Mean (SE) & $\begin{array}{r}\text { Change } \\
\text { from } \\
\text { baseline }\end{array}$ & p-Value & Mean (SE) & $\begin{array}{r}\text { Change } \\
\text { from } \\
\text { baseline }\end{array}$ & p-Value & & \\
\hline \multirow[t]{8}{*}{ MCA } & PSV & Before & $58.8(2.5)$ & & & $66.7(2.5)$ & & & 0.01 & \\
\hline & & Immediately after & $63.9(2.1)$ & 5.1 & 0.006 & $63.5(2.1)$ & -3.2 & 0.08 & 0.88 & 0.001 \\
\hline & & 5 minutes after & $64.2(1.8)$ & 5.4 & 0.001 & $63.7(1.8)$ & -3.0 & 0.07 & 0.80 & $<0.001$ \\
\hline & & 10 minutes after & $64.8(2.0)$ & 6.0 & $<0.001$ & $63.6(2.0)$ & -3.1 & 0.05 & 0.59 & $<0.001$ \\
\hline & EDV & Before & $27.3(1.0)$ & & & $31.2(1.0)$ & & & 0.008 & \\
\hline & & Immediately after & $34.7(1.1)$ & 7.4 & $<0.001$ & $29.6(1.1)$ & -1.6 & 0.14 & 0.001 & $<0.001$ \\
\hline & & 5 minutes after & $37.0(0.8)$ & 9.7 & $<0.001$ & $29.8(0.8)$ & -1.5 & 0.12 & $<0.001$ & $<0.001$ \\
\hline & & 10 minutes after & $37.9(1.0)$ & 10.6 & $<0.001$ & $29.3(1.0)$ & -2.0 & 0.03 & $<0.001$ & $<0.001$ \\
\hline \multirow[t]{8}{*}{ ICA } & PSV & Before & $71.3(2.8)$ & & & $69.4(2.8)$ & & & 0.39 & \\
\hline & & Immediately after & $69.3(2.5)$ & -2.0 & 0.27 & $64.3(2.5)$ & -5.1 & 0.005 & 0.09 & 0.18 \\
\hline & & 5 minutes after & $68.8(2.4)$ & -2.5 & 0.15 & $63.8(2.4)$ & -5.6 & 0.002 & 0.07 & 0.17 \\
\hline & & 10 minutes after & $69.0(2.3)$ & -2.2 & 0.21 & $64.2(2.3)$ & -5.2 & 0.004 & 0.06 & 0.21 \\
\hline & EDV & Before & $26.5(0.9)$ & & & $26.3(0.9)$ & & & 0.81 & \\
\hline & & Immediately after & $29.0(1.0)$ & 2.5 & $<0.001$ & $24.7(1.0)$ & -1.6 & 0.02 & $<0.001$ & $<0.001$ \\
\hline & & 5 minutes after & $30.6(0.9)$ & 4.2 & $<0.001$ & $24.8(0.9)$ & -1.5 & 0.02 & $<0.001$ & $<0.001$ \\
\hline & & 10 minutes after & $31.9(0.9)$ & 5.4 & $<0.001$ & $24.3(0.9)$ & -1.9 & 0.006 & $<0.001$ & $<0.001$ \\
\hline \multirow[t]{8}{*}{ VA } & PSV & Before & $42.1(1.5)$ & & & $39.7(1.5)$ & & & 0.24 & \\
\hline & & Immediately after & $43.6(1.7)$ & 1.5 & 0.21 & $39.1(1.7)$ & -0.6 & 0.61 & 0.04 & 0.22 \\
\hline & & 5 minutes after & $44.0(1.6)$ & 2.0 & 0.12 & $38.7(1.6)$ & -1.0 & 0.43 & 0.005 & 0.10 \\
\hline & & 10 minutes after & $43.3(1.6)$ & 1.3 & 0.31 & $37.9(1.6)$ & -1.8 & 0.16 & 0.005 & 0.09 \\
\hline & EDV & Before & $14.7(0.6)$ & & & $14.4(0.6)$ & & & 0.63 & \\
\hline & & Immediately after & $18.0(0.7)$ & 3.3 & $<0.001$ & $13.9(0.7)$ & -0.6 & 0.19 & $<0.001$ & $<0.001$ \\
\hline & & 5 minutes after & $19.3(0.7)$ & 4.6 & $<0.001$ & $13.6(0.7)$ & -0.9 & 0.09 & $<0.001$ & $<0.001$ \\
\hline & & 10 minutes after & $19.8(0.7)$ & 5.0 & $<0.001$ & $13.2(0.7)$ & -1.2 & 0.01 & $<0.001$ & $<0.001$ \\
\hline
\end{tabular}

EDV, end-diastolic velocity; ICA, internal carotid artery; MCA, middle cerebral artery; OAD, occipitoatlantal decompression; PSV, peak systolic velocity; SE, standard error; VA, vertebral artery. 
timepoints and for changes from baseline to each posttreatment timepoint after OAD were greater in the MCA, ICA, and VA than after sham touch (all $\mathrm{p}<0.001$ ).

PSV in the MCA showed an interaction between time and treatment where differences between treatments existed only at baseline $(\mathrm{p}<0.001$; Table 1$)$. The baseline PSV in the MCA was lower for OAD (mean [SE], 58.8 [2.5]) than sham touch (66.7 [2.5]; p=0.01); however, PSV increased immediately after OAD (63.9 [2.1]; difference, 5.1), reaching similar levels as those after sham touch at all post-treatment timepoints (all $\mathrm{p}>0.59$; Table 1 ). For PSV in the ICA and VA, there were no interactions between time and treatment (both $\mathrm{p}>0.35$ ). PSV in the ICA decreased from baseline (69.4 [2.8]) to immediately after sham touch (64.3 [2.5]; difference $-5.1 ; \mathrm{p}=0.005$ ), but this decrease was not different from the nominal decrease observed immediately after OAD (71.3 [2.8] to 69.3 [2.5]; difference $-2.0 ; p=0.18)$. Overall, there were no changes over time for PSV in the VA ( $\mathrm{p}=0.54)$.

Changes over time did not depend on treatment for BP or HR (all $p>0.06$ ), and there were no changes over time (all $\mathrm{p}>0.30)$.

\section{Discussion}

The current study evaluated the effects of OAD on intracranial and extracranial blood flow dynamics. Blood flow parameters (PSV and EDV) were assessed with Doppler ultrasonography, while the systemic effect of OAD was assessed using HR and BP. The brain is supplied by branches of the circle of Willis, and the circle itself is formed by the left and right ICA and VA. As such, blood supply to the brain depends entirely on these four arteries. ${ }^{37}$ Further, the left and right MCA are two of the main cerebral arteries that perfuse the brain. ${ }^{38}$ Thus, significant changes in blood flow parameters in the MCA, ICA, and VA would result in changes in perfusion of the brain.

Our results showed that diastolic flow (based on the EDV) increased in the MCA, ICA, and VA immediately after $\mathrm{OAD}$ and for at least 10 minutes after when compared with our sham touch treatment intervention. This effect has been caused by decreased resistance to flow, allowing greater flow during diastole. Since flow resistance $(R)$ is inversely proportional to vessel radius $(r)$ raised to the fourth power $\left(R=8 \mathrm{Ln} / \pi r^{4}\right.$; where $L=$ vessel length and $n$ = blood viscosity), even a slight increase in vessel diameter from decreased external tissue pressure could lead to significant changes in resistance and accompanying blood flow. ${ }^{39}$ The increase in flow through the ICA and VA continued through the circle of Willis and into the cerebral circulation, leading to the increased diastolic flow of the MCA during the 10 minutes after OAD and, thus, improving perfusion of the brain. The exact mechanism of these changes is unknown but could be explained by either parasympathetic stimulation through the secretion of vasodilating neurotransmitters such as calcitonin generelated peptide or nitric oxide ${ }^{40}$ or by a decrease in external tissue pressure on ICA and VA with the resulting flow causing further dilation in the MCA.

Other studies have reported changes in vascular resistance in the carotids with the activation of the trigeminoparasympathetic reflex. ${ }^{41}$ The skin over the occiput and upper neck is innervated by the sensory branches of C2. It is not part of the trigeminal complex, but studies ${ }^{42,43}$ have shown possible functional connectivity between the trigeminal and occipital nerves indicating that trigeminal nerve can receive sensory information from the greater occipital nerve and branches of the upper cervical roots. This means that the trigeminoparasympathetic reflex can be activated through the greater occipital nerve and sensory branches of the $\mathrm{C} 2$. The use of sham touch treatment intervention in the current study allowed us to control for the possible effect of autonomic activation due to simple skin touch, but OAD would have involved the stimulation of the deeper nerves possibly leading to the increased parasympathetic tone. Other studies showed autonomic activation (both sympathetic and parasympathetic) after cervical adjustment. ${ }^{44}$ Therefore, it is possible that OAD triggered an autonomic response that led to a decrease in vascular resistance in the ICA and MCA, but did not cause changes in HR, BP, or systolic flow.

These results may prove useful for better treating patients with headache disorders, whether for the pain symptom itself or other physiological manifestations. A systematic review ${ }^{45}$ of patients with migraines found decreased flow velocity in the MCA in the early phases of spontaneous migraine attacks. A brief review ${ }^{46}$ showed that flow was dynamic during different phases of migraines, primarily during migraine with aura. During the aura phase, there were decreases in cerebral blood flow in the posterior cerebral circulation and moved anteriorly. Migraine without aura and the interictal periods for migraineurs with or without aura do not have as many notable cerebral flow changes. For the interictal periods, the flow appears similar to healthy patients. ${ }^{46}$ A 2017 study ${ }^{47}$ investigating different clinical courses of migraine found decreases in basilar artery flow and increases in posterior cerebral artery flow when patients with chronic migraine changed to episodic migraine $(<15$ attacks/ month) but no blood flow changes when chronic migraine persisted ( $\geq 15$ attacks/month). The same study found that 
patients with episodic migraine in remission had decreased MCA and basilar artery flow. ${ }^{47}$ Taken together, these studies ${ }^{45-47}$ suggest that there are distinctive changes in cerebral blood flow for different types of migraines, in different arteries, and at different timepoints. Thus, given the results of our study, treatment with OAD may be most beneficial for patients when more blood flow is needed in the cerebral vasculature during certain migraine attacks or at specific timepoints during the progression of those attacks.

Limitations of the current study include a small sample size and the use of young, healthy participants. However, we specifically included first-year osteopathic medical students as our study population because they had not yet been exposed to cervical and cranial osteopathic manipulative techniques. As such, they were less likely to know whether the $\mathrm{OAD}$ technique or the sham touch treatment was performed. Our use of healthy participants may limit the generalizability of our results. Therefore, future studies could expand this research by using a similar methodology as the current study while recruiting patients with various known headache disorders (e.g., migraine, tension-type, cluster). The addition of a subjective pain assessment in such studies may also be beneficial. Finally, given that research has shown a variety of hemodynamic patterns in different types of migraines, studies that record the type of migraine experienced by each patient may be useful to understand the physiological effects of OAD treatment further when applied to migraine-type headache disorders.

\section{Conclusion}

This randomized, single-blinded, crossover study evaluated blood flow in the intracranial and extracranial vasculature before and after OAD using Doppler ultrasonography. Our results suggested an increase in diastolic flow in the MCA, ICA, and VA after OAD when compared with a sham touch treatment intervention. It seems likely that OAD decreased vascular resistance increasing blood flow through the circle of Willis, which resulted in better brain perfusion as evidenced by the increased MCA flow. The exact mechanism of this increase is unknown, but can be explained by either parasympathetic stimulation through the secretion of vasodilating neurotransmitters or by decrease in external tissue pressure on ICA and VA with the resulting flow causing further dilation in the MCA. As research on headache disorders evolves, the current study may help physicians better understand how to use OAD to target specific phases or symptoms during the clinical course of migraines or other headache disorders to establish hemodynamic balance and improve outcomes. In more general terms, our results may also establish physiological evidence for the theory that OMT can improve circulation.

Acknowledgments: The authors thank Deborah Goggin, MA, ELS, from the Department of Research Support at A.T. Still University, for help with manuscript preparation.

Research funding: None declared.

Author contributions: All authors provided substantial contributions to conception and design, acquisition of data, or analysis and interpretation of data; all authors drafted the article or revised it critically for important intellectual content; all authors gave final approval of the version of the article to be published; and all authors agree to be accountable for all aspects of the work in ensuring that questions related to the accuracy or integrity of any part of the work are appropriately investigated and resolved.

Competing interests: Authors state no conflict of interest. Ethical approval: Research involving human subjects has been approved by the authors' Institutional Review Board (A.T. Still University), but was not included in a clinical trial registry according to Declaration of Helsinki requirements.

\section{References}

1. DeStefano LA. Greenman's principles of manual medicine: Lippincott Williams \& Wilkins; 2017.

2. Kimberly PE, Dickey J, Halma KD. Outline of Osteopathic Manipulative Procedures: The Kimberly Manual. Marceline, MO: Walsworth; 2008.

3. Seffinger MA, Hruby RJ, Rogers FJ, et al. Philosophy of osteopathic medicine. In: Seffinger MA, ed. Foundations of Osteopathic Medicine: Philosophy, Science, Clinical Applications, and Research. 4th ed. Philadelphia, PA: Wolters Kluwer; 2018:2-18.

4. Pierce-Talsma S, Peña N. Condylar decompression technique for infants. J Am Osteopath Assoc. 2017;117(11):e136. doi: 10.7556/ jaoa.2017.139.

5. Lund GC, Edwards G, Medlin B, Keller D, Beck B, Carreiro JE. Osteopathic manipulative treatment for the treatment of hospitalized premature infants with nipple feeding dysfunction. J Am Osteopath Assoc. 2011;111(1):44-48. doi: 10.7556/jaoa.2011. 111.1.44.

6. Giles PD, Hensel KL, Pacchia CF, Smith ML. Suboccipital decompression enhances heart rate variability indices of cardiac control in healthy subjects. J Altern Complement Med. 2013;19(2): 92-96. doi: 10.1089/acm.2011.0031.

7. Cerritelli F, Ginevri L, Messi G, et al. Clinical effectiveness of osteopathic treatment in chronic migraine: 3-Armed randomized controlled trial. Complement Ther Med. 2015;23(2):149-156. doi: 10.1016/j.ctim.2015.01.011. 
8. Voigt K, Liebnitzky J, Burmeister U, et al. Efficacy of osteopathic manipulative treatment of female patients with migraine: results of a randomized controlled trial. J Altern Complement Med. 2011; 17(3):225-230. doi: 10.1089/acm.2009.0673.

9. Shi X, Rehrer S, Prajapati P, Stoll ST, Gamber RG, Downey HF. Effect of cranial osteopathic manipulative medicine on cerebral tissue oxygenation. J Am Osteopath Assoc. 2011;111(12):660-666.

10. Anderson RE, Seniscal C. A comparison of selected osteopathic treatment and relaxation for tension-type headaches. Headache. 2006;46(8):1273-1280. doi: 10.1111/j.1526-4610.2006.00535.x.

11. Tamburella F, Piras F, Piras F, Spanò B, Tramontano M, Gili T. Cerebral perfusion changes after osteopathic manipulative treatment: a randomized manual placebo-controlled trial. Front Physiol. 2019;10:403. doi: 10.3389/fphys.2019.00403.

12. Cerritelli F, Lacorte E, Ruffini N, Vanacore N. Osteopathy for primary headache patients: a systematic review. J Pain Res. 2017; 10:601. doi: 10.2147/JPR.S130501.

13. Cerritelli F, Ruffini N, Lacorte E, Vanacore N. Osteopathic manipulative treatment in neurological diseases: systematic review of the literature. J Neurol Sci. 2016;369:333-341. doi: 10.1016/j.jns.2016.08.062.

14. Digre KB. Headaches and other head pain. In: Goldman L, Schafer Al, eds. Goldman's Cecil Medicine. 24th ed. Philadelphia, PA: Elsevier; 2012:2246-2252.

15. van Hoogstraten WS, MaassenVanDenBrink A. The need for new acutely acting antimigraine drugs: moving safely outside acute medication overuse. J Headache Pain. 2019;20(1):54. doi: 10.1186/s10194-019-1007-y.

16. Lopez D, King HH, Knebl JA, Kosmopoulos V, Collins D, Patterson RM. Effects of comprehensive osteopathic manipulative treatment on balance in elderly patients: a pilot study. J Am Osteopath Assoc. 2011;111(6):382-388. doi: 10.7556/ jaoa.2011.111.6.382.

17. Jacobs B, Dussor G. Neurovascular contributions to migraine: moving beyond vasodilation. Neuroscience. 2016;338:130-144. doi: 10.1016/j.neuroscience.2016.06.012.

18. Fabjan A, Zaletel M, Žvan B. Is there a persistent dysfunction of neurovascular coupling in migraine? Biomed Res Int. 2015;2015: 574186. doi: $10.1155 / 2015 / 574186$.

19. Goadsby PJ. The vascular theory of migraine: a great story wrecked by the facts. Brain. 2009;132(1):6-7. doi: 10.1093/brain/ awn321.

20. Brennan KC, Charles A. An update on the blood vessel in migraine. Curr Opin Neurol. 2010;23(3):266-274. doi: 10.1097/ WCO.0b013e32833821c1.

21. Noseda R, Burstein R. Migraine pathophysiology: anatomy of the trigeminovascular pathway and associated neurological symptoms, cortical spreading depression, sensitization, and modulation of pain. Pain. 2013;154(suppl 1):S44-S53. doi: 10. 1016/j.pain.2013.07.021.

22. Headache Classification Committee of the International Headache Society (IHS). The International Classification of Headache Disorders, 3rd edition. Cephalalgia. 2018;38(1):1-211. doi: $10.1177 / 0333102417738202$.

23. Jameson JL. Harrison's manual of medicine, 20th edition. 20th ed; 2018.

24. Durham PL. Calcitonin gene-related peptide (CGRP) and migraine. Headache. 2006;46:S3-S8. doi: 10.1111/j.1526-4610.2006. 00483.x.
25. Durham PL, Vause CV. Calcitonin gene-related peptide (CGRP) receptor antagonists in the treatment of migraine. CNS drugs. 2010;24(7):539-548. doi: 10.2165/11534920-000000000-00000.

26. Dodick DW. A Phase-by-Phase Review of Migraine Pathophysiology. Headache. 2018;58 Suppl 1:4-16. doi: 10.1111/ head.13300.

27. Noseda R, Burstein R. Migraine pathophysiology: anatomy of the trigeminovascular pathway and associated neurological symptoms, cortical spreading depression, sensitization, and modulation of pain. Pain. 2013;154:S44-S53. doi: 10.1016/j.pain. 2013.07.021.

28. Pietrobon D, Moskowitz MA. Pathophysiology of migraine. Annu Rev Physiol. 2013;75:365-391. doi: 10.1146/annurev-physiol030212-183717.

29. Gallagher RM. Headache pain. J Am Osteopath Assoc. 2005;105(9 suppl 4):S7-S11.

30. Zanette EM, Agnoli A, Roberti C, Chiarotti F, Cerbo R, Fieschi C. Transcranial Doppler in spontaneous attacks of migraine. Stroke. 1992;23(5):680-685. doi: 10.1161/01.str.23.5.680.

31. Viola S, Viola P, Litterio P, Buongarzone MP, Fiorelli L. Pathophysiology of migraine attack with prolonged aura revealed by transcranial Doppler and near infrared spectroscopy. Neurol Sci. 2010;31(suppl 1):S165-166. doi: 10.1007/s10072-010-0318-1.

32. Kaufmann H. Mechanisms, causes, and evaluation of orthostatic hypotension. UpToDate Web site. https://www.uptodate.com/ contents/mechanisms-causes-and-evaluation-of-orthostatichypotension\#H35622970. Updated May 28, 2019. Accessed April 14, 2020.

33. Freeman R, Wieling W, Axelrod FB, et al. Consensus statement on the definition of orthostatic hypotension, neurally mediated syncope and the postural tachycardia syndrome. Auton Neurosci. 2011;161(1-2):46-48. doi: 10.1016/j.autneu.2011.02.004.

34. Arnold AC, Shibao C. Current concepts in orthostatic hypotension management. Curr Hypertens Rep. 2013;15(4):304-312. doi: 10. 1007/s11906-013-0362-3.

35. Standring S, ed. Gray's Anatomy: The Anatomical Basis of Clinical Practice. 41st ed. London, UK: Elsevier; 2016.

36. Moore KL, Dalley AF, Agur AM. Clinically Oriented Anatomy. 7th ed. Baltimore, MD: Lippincott Williams \& Wilkins; 2014.

37. Vrselja Z, Brkic H, Mrdenovic S, Radic R, Curic G. Function of circle of Willis. J Cereb Blood Flow Metab. 2014;34(4):578-584. doi: 10. 1038/jcbfm.2014.7.

38. Cilliers K, Page BJ. Anatomy of the middle cerebral artery: cortical branches, branching pattern and anomalies. Turk Neurosurg. 2017;27(5):671-681. doi: 10.5137/1019-5149.JTN.18127-16.1.

39. Delong C, Sharma S. Physiology, Peripheral Vascular Resistance. Treasure Island, FL: StatPearls Publishing; 2019.

40. Sheng Y, Zhu L. The crosstalk between autonomic nervous system and blood vessels. Int J Physiol Pathophysiol Pharmacol. 2018; 10(1):17-28.

41. Avnon Y, Nitzan M, Sprecher E, Rogowski Z, Yarnitsky D. Different patterns of parasympathetic activation in uni- and bilateral migraineurs. Brain. 2003;126(7):1660-1670. doi: 10.1093/brain/ awg158.

42. Busch V, Jakob W, Juergens T, Schulte-Mattler W, Kaube H, May A. Functional connectivity between trigeminal and occipital nerves revealed by occipital nerve blockade and nociceptive blink reflexes. Cephalalgia. 2006;26(1):50-55. doi: 10.1111/j.14682982.2005.00992.x. 
43. Piovesan EJ, Kowacs PA, Oshinsky ML. Convergence of cervical and trigeminal sensory afferents. Curr Pain Headache Rep. 2003; 7(5):377-383. doi: 10.1007/s11916-003-0037-x.

44. Welch A, Boone R. Sympathetic and parasympathetic responses to specific diversified adjustments to chiropractic vertebral subluxations of the cervical and thoracic spine. J Chiropr Med. 2008;7(3):86-93. doi: 10.1016/j.jcm.2008.04.001.

45. Shayestagul NA, Christensen CE, Amin FM, Ashina S, Ashina M. Measurement of blood flow velocity in the middle cerebral artery during spontaneous migraine attacks: a systematic review. Headache. 2017;57(6):852-861. doi: 10.1111/head.13106.

46. Bartolini M, Baruffaldi R, Paolino I, Silvestrini M. Cerebral blood flow changes in the different phases of migraine. Funct Neurol. 2005;20(4):209-211.

47. Lee MJ, Chu MK, Choi H, Choi HA, Lee C, Chung CS. Longitudinal changes in cerebral blood flow velocities in different clinical courses of migraine. Cephalalgia. 2017;37(10):927-937. doi: 10. $1177 / 0333102416658715$. 